\title{
Report on Acute Aortic Dissection Type A
}

\author{
C. Simoglou, F. Konstantinou, D. Mikroulis, S. Eleftheriadis, G. Bougioukas \\ Department of Cardiothoracic Surgery, University General Hospital of Evros, Democritus University of Thrace, \\ Alexandroupolis, Greece \\ Email: simoglouchristosdoctor@yahoo.com
}

Received 10 November 2014; accepted 10 January 2015; published 14 January 2015

Copyright (C) 2015 by authors and Scientific Research Publishing Inc.

This work is licensed under the Creative Commons Attribution International License (CC BY).

http://creativecommons.org/licenses/by/4.0/

(c) (i) Open Access

\section{Abstract}

Background: Acute aortic dissection is a common life-threatening disorder affecting the aorta. The immediate mortality rate in aortic dissection is as high as $\mathbf{1 \%}$ per hour over the first several hours, making early diagnosis and treatment critical for survival. Case presentation: We are presenting a case of Stanford Type A aortic dissection in a 58-year-old male patient with a history of hypertension. He arrived at the emergency department (ED) with diagnosed acute coronary syndrome a few hours after a sudden and severe worsening of his epigastric pain. Interesting case where the dissection starts from the orifice of the right coronary artery, occupies the aortic valve. Conclusion: Predictors of follow-up this cause mortality reflect patient history variables as opposed to in-hospital parameters or in-hospital complications, which may be explained by the successful in-hospital treatment of the acute dissection.

\section{Keywords}

Stanford A, Aneurysm, Cardiovascular Diseases, Marfan's Syndrome, De Bakey Classification

\section{Introduction}

Aortic dissection is one of the acute aortic syndromes and a type of arterial dissection. It occurs when blood enters the medial layer of the aortic wall through a tear or penetrating ulcer in the intima and tracks along the media, forming a second blood-filled channel within the wall [1]. The main causes of dissection are hypertension, atherosclerosis, Marfan's syndrome, Ehlers-Danlos syndrome, vasculitis, pregnancy and iatrogenic (aortic catheterization) [2]. The dissection of the layers of the aortic wall produces a false lumen, which spirals throughout a portion or commonly, the entire length of the aorta. In the United States, the prevalence of aortic dissection ranges from 0.2 to 0.8 per 100,000 per year or roughly 2000 to 3000 new cases per year [3]. 


\section{Complications}

Complications of aortic dissection include dissection and occlusion of branch vessels, distal thromboembolism, aneurismal dilatation and aortic rupture. Type A dissection may also result in coronary artery occlusion, aortic regurgitation and pericardial tamponade and therefore management of this type of dissection is usually emergency surgical repair [4]. Type B dissections are usually managed with aggressive blood pressure control unless there are complications. An acute aortic dissection usually causes a severe sharp, tearing pain in the chest and upper back. In certain patients, the pain may develop or concentrate in the abdomen. In a very small number of patients, they may have little to no symptoms or flu-like symptoms. The weakening of the aortic wall can lead to aortic rupture within minutes or hours of the acute event. The presence of the false channel in the ascending aorta can result in regurgitation of the aortic valve and myocardial infarction (heart attack) [5]. If the disturbance of aortic valve is significant this can lead to sudden symptoms of heart failure secondary to severe aortic valve regurgitation.

\section{Case Presentation}

The patient is a 58-year-old male with a past medical history of hypertension. He arrived at emergency department (ED) with diagnosis acute coronary syndrome a few hours after he developed sudden, severe worsening of his epigastric pain. The pain was stabbing, radiating to the back and was associated with nausea and profuse sweating. Shortly after to the ED, he complained of pain extending to his chest. On physical examination his blood pressure was 160/80 MmHg and pulse 98 beats per minute. He had tenderness to, especially in the epigastrium with associated guarding. The chest, lung, and cardiac exams were reported as normal, the remainder of the physical examination was unremarkable. Lab results were all within normal limits. Fast transesophageal echocardiogram was performed (Figure 1). Electrocardiogram showed sinus tachycardia with acute ischemic changes. A chest CT verified the origin of the dissection at the aortic root. Image aortic dissection, Type A which extends from the origin of the aortic root, dissection the right-hand valsava mouth of bay and the threefold aortic valve, where the separation at this stage reaches the abdominal aorta (at the level below about $2 \mathrm{~cm}$ of neurite renal arteries), expands the origin of the right artery anonymous and origin of the left subclavian artery. Possible extension of the dissection is in the left medial arteria carotids (Figure 2(a) and Figure 2(b)).

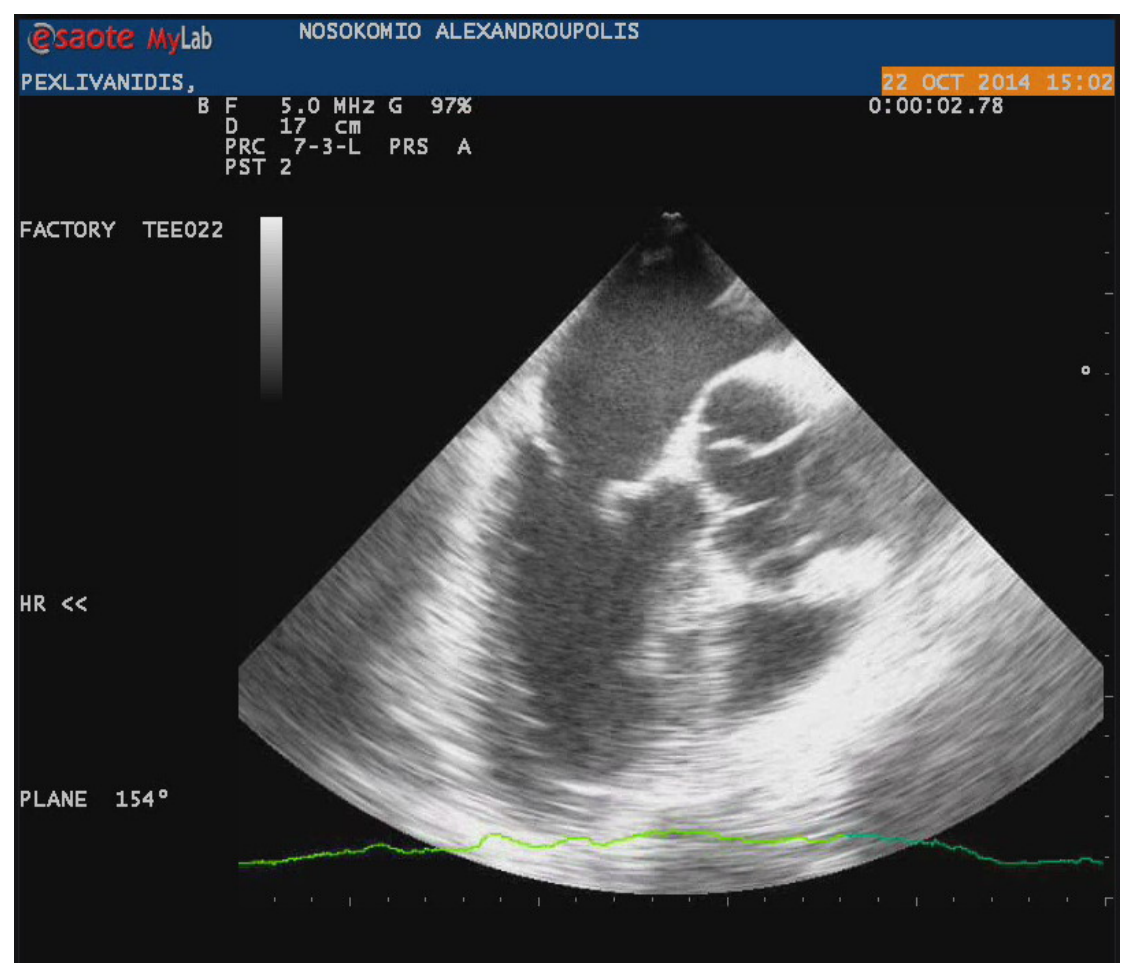

Figure 1. Transesophageal echocardiogram revealing intimal flap in ascending aorta. 
It also marked aneurismal dilatation of the aortic root and mild dilatation of the aortic arch. The patient was started on intravenous Esmolol and nitroprusside. Immediately after the patient was transferred to Aortography (Figure 3(a) and Figure 3(b)). The cardiothoracic surgery team was consulted and the patient was taken to the operating room emergently, a Bentall operation was performed.

\section{Diagnosis}

The diagnosis of a Type A aortic dissection is primarily based on clinical symptoms and radiographic imaging studies. The chest X-ray may provide the first suggestion of thoracic aortic pathology. The X-ray may show a pronounced heart shadow or silhouette indicating possible aortic aneurysm or aortic rupture. Obviously, the chest X-ray may tell the physician that another medical condition is causing the symptoms. An excellent study to diagnose an aortic dissection and is accessible in most emergency departments is a CT scan (or CT angiogram) MRI/A is also an excellent radiographic study, however, it is less accessible, more time consuming, and less patient-friendly [6]. A transesophageal echocardiogram (TEE) is a very good study to diagnosis an aortic dissection, but it requires local and iv anesthesia AND is also less accessible and patient-friendly.

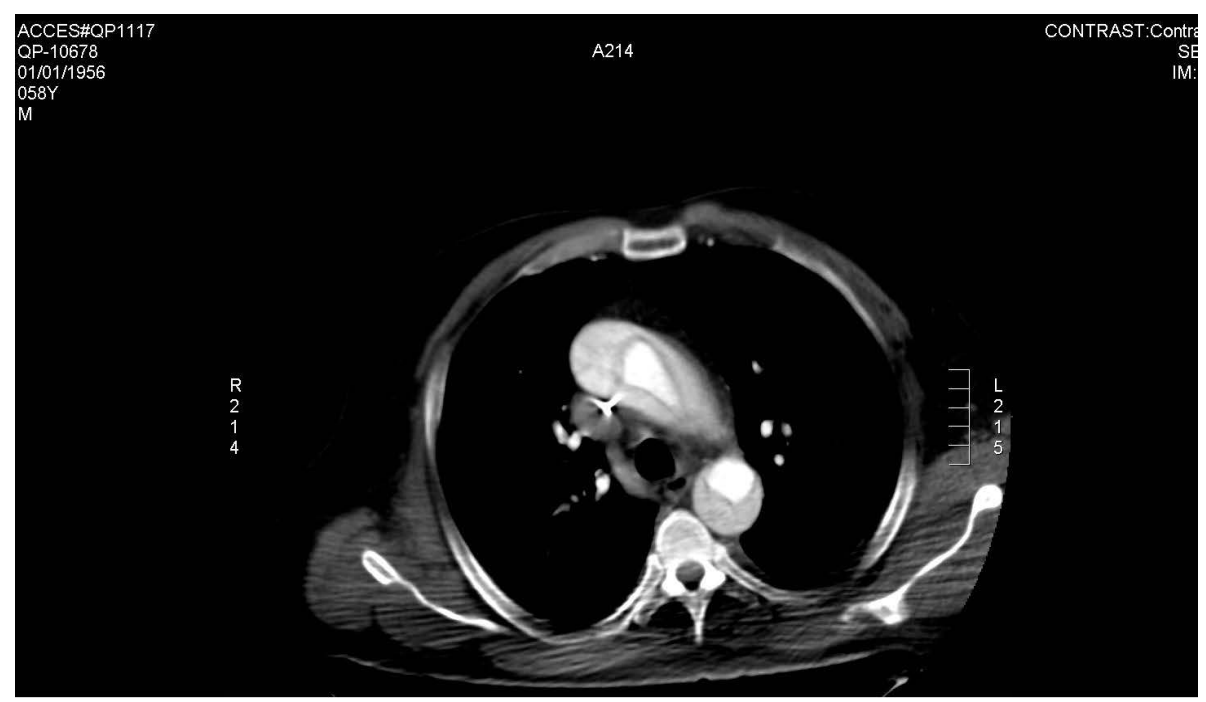

(a)

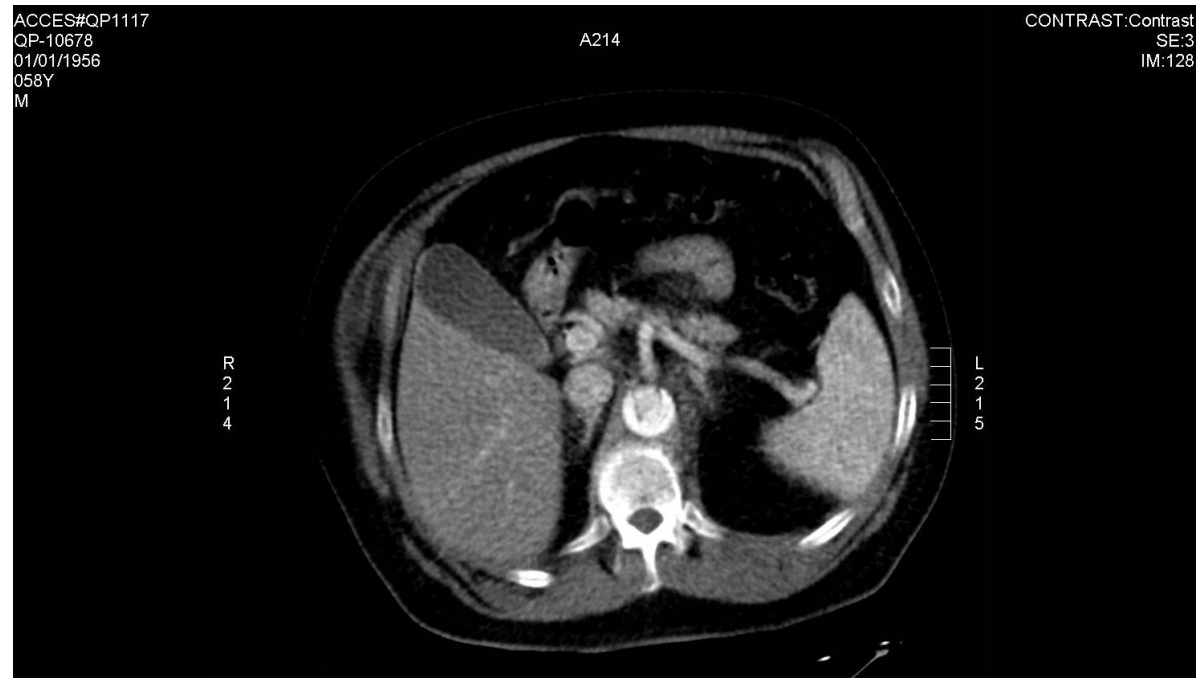

(b)

Figure 2. CT scans done on admission day. Notice the aortic intimal flap in ascending aorta, descending thoracic aorta. 
A blood test that may increase the suspicion of an acute aortic dissection is D-dimer. However, D-dimer is elevated in other medical conditions. Some studies have suggested that if the D-dimer is above a certain level the likelihood of an aortic dissection is greater than $90 \%$ especially if the test is performed within 6 hours of onset of symptoms. As the time interval increases to 24 hours the sensitive and specificity diminishes significantly. At this point, D-dimer can be used to stratify patients with symptoms suggestive of acute dissection to further imaging studies or continued observation.

\section{Treatment}

The treatment of aortic dissection depends on the part of the aorta involved. Surgery is usually required for dissections that involve the aortic arch, while dissections of the part further away from the heart may be treated with blood pressure lowering only.

\section{Discussion}

Once the acute dissection occurs, the mortality increases $1 \%$ per hour until patient has aortic repair. Most

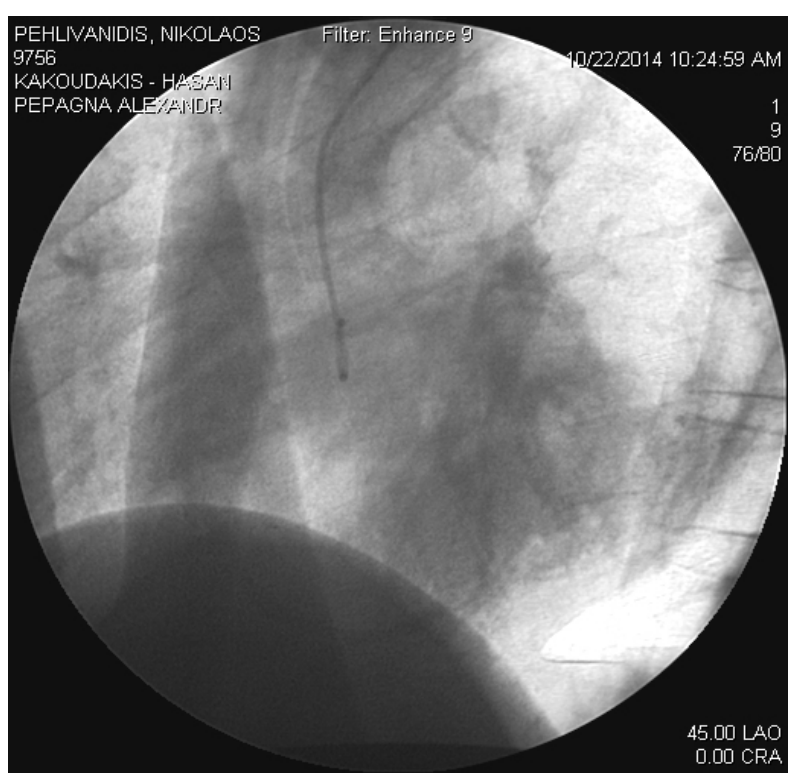

(a)

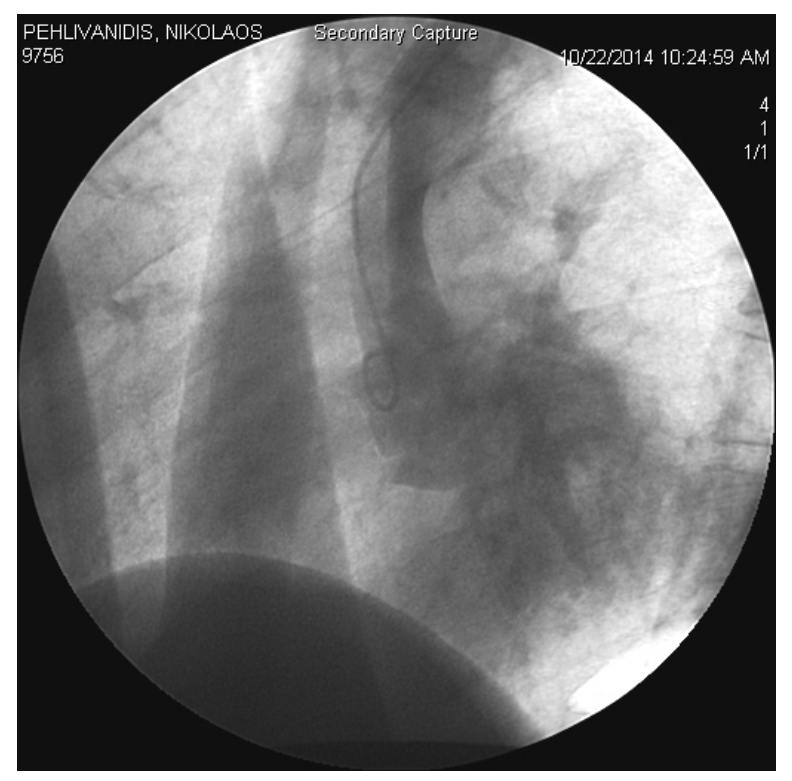

(b)

Figure 3. Aortography done on admission day revealing intimal flap in ascending aorta.

Table 1. Stanford and De Bakey classification.

(a)

Stanford Classification

Type Characteristic

Type A Dissection involving the ascending aorta, regardless of the site of the primary tear

Type B Dissection of the descending aorta

(b)

De Bakey Classification

\section{Type Characteristic}

Type 1 Dissection of the ascending and descending thoracic aorta

Type 2 Dissection of the ascending aorta

Type 3 Dissection of the descending aorta 
patients that develop a Type A aortic dissection have a history of elevated blood pressure, an ascending aortic aneurysm, connective tissue disorder, bicuspid aortic valve, or have endured a stressful/emotional life event. Based on the results of this study, TEE may play an increasingly important role in patients with acute Type A aortic dissection as cardiologists and cardiac surgeons work together to determine the optimal management of any consequent AR (Table $1(a)$ ).

Aortography was previously considered the gold standard test for diagnosis. Aortography identifies the false and true lumens, assesses involvement of the arch vessels, and detects aortic valve insufficiency [7]. However, Aortography is also an invasive, time-consuming technique that requires the use of potentially nephroxic contrast. The European cooperative study demonstrated that the diagnostic accuracy of Aortography is not as high as originally thought, with sensitivity of $88 \%$ and specificity of $94 \%$. Under diagnosis of aortic dissection with Aortography can be due to thrombosis of the false lumen or the simultaneous operation of both the false and true lumens [8]. Noninvasive imaging modalities, such as spiral CT multiplaner transesophageal echocardiogram (TEE), and magnetic resonance imaging (MRI) are replacing Aortography for purpose of evaluating for aortic dissection (Table $1(b))$.

\section{References}

[1] Knaut, A.L. and Cleveland, J.C. (2003) Aortic Emergencies. Emergency Medicine Clinics of North America, 21, 817845. http://dx.doi.org/10.1016/S0733-8627(03)00063-4

[2] Rushid, J., Eisenberg, M.J. and Topol, E.J. (1996) Cocaine-Indueed Aortic Dissection. American Heart Journal, 132, 1301-1304. http://dx.doi.org/10.1016/S0002-8703(96)90486-X

[3] Nienaber, C.A., von Kodolitsh, Y. and Nikolas, V. (1993) The Diagnosis of Thoracic Aortic Dissection by Noninvasive Imaging Procrdures. The New England Journal of Medicine, 328, 1-9. http://dx.doi.org/10.1056/NEJM199301073280101

[4] Vasile, N., Mathieu, D. and Keita, K. (1986) Computed Tomography of Thoracic Aortic Dissection: Accuracy and Pitfalls. Journal of Computer Assisted Tomography, 10, 211-215. http://dx.doi.org/10.1097/00004728-198603000-00008

[5] Kawahito, K., Adachi, H., Yamaguchi, A. and Ino, T. (2001) Preoperative Risk Factors for Hospital Mortality in Acute Type A Aotic Dissection. The Annals of Thoracic Surgery, 71, 1239-1243. http://dx.doi.org/10.1016/S0003-4975(00)02654-0

[6] Fleming, C., Whitlock, E.P., Beil, T.L. and Lederle, F.A. (2005) Screening for Abdominal Aortic Aneurysm: A BestEvidence Systematic Review for the US Preventive Services Task Force. Annals of Internal Medicine, 142, 203-211. http://dx.doi.org/10.7326/0003-4819-142-3-200502010-00012

[7] Liao, M.F., Jing, Z.P. and Bao, J.M. (2006) Role of Nitrix Oxide and Inducible Nitrix Oxide Synthase in Human Abdominal Aortic Aneurysms: A Preliminary Study. Chinese Medical Journal, 119, 312.

[8] Tsai, T.T., Nienaber, C.A. and Eagle, K.A. (2005) Acute Aortic Syndromes. Circulation, 112, 3802-3813. http://dx.doi.org/10.1161/CIRCULATIONAHA.105.534198 
Scientific Research Publishing (SCIRP) is one of the largest Open Access journal publishers. It is currently publishing more than 200 open access, online, peer-reviewed journals covering a wide range of academic disciplines. SCIRP serves the worldwide academic communities and contributes to the progress and application of science with its publication.

Other selected journals from SCIRP are listed as below. Submit your manuscript to us via either submit@scirp.org or Online Submission Portal.
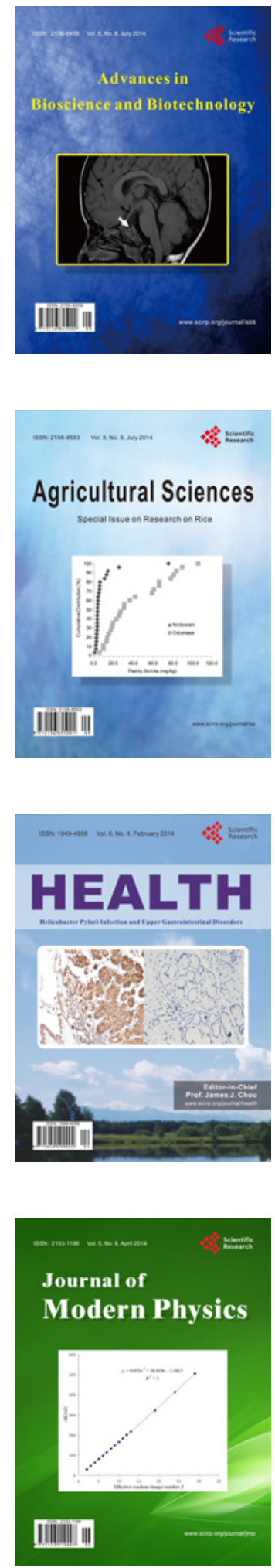
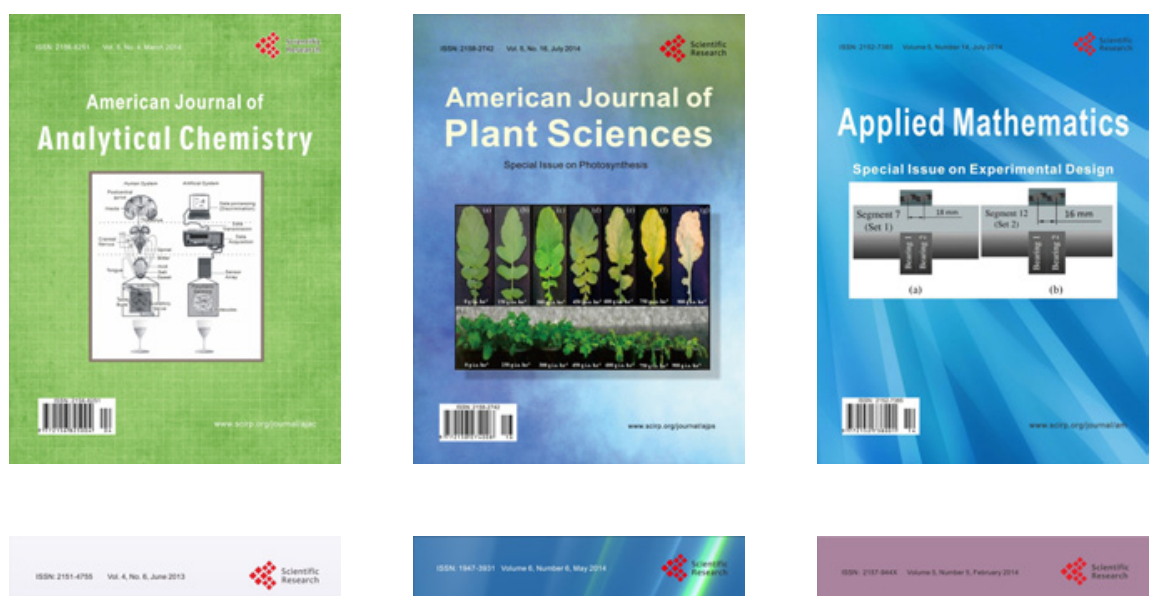

Creative Education
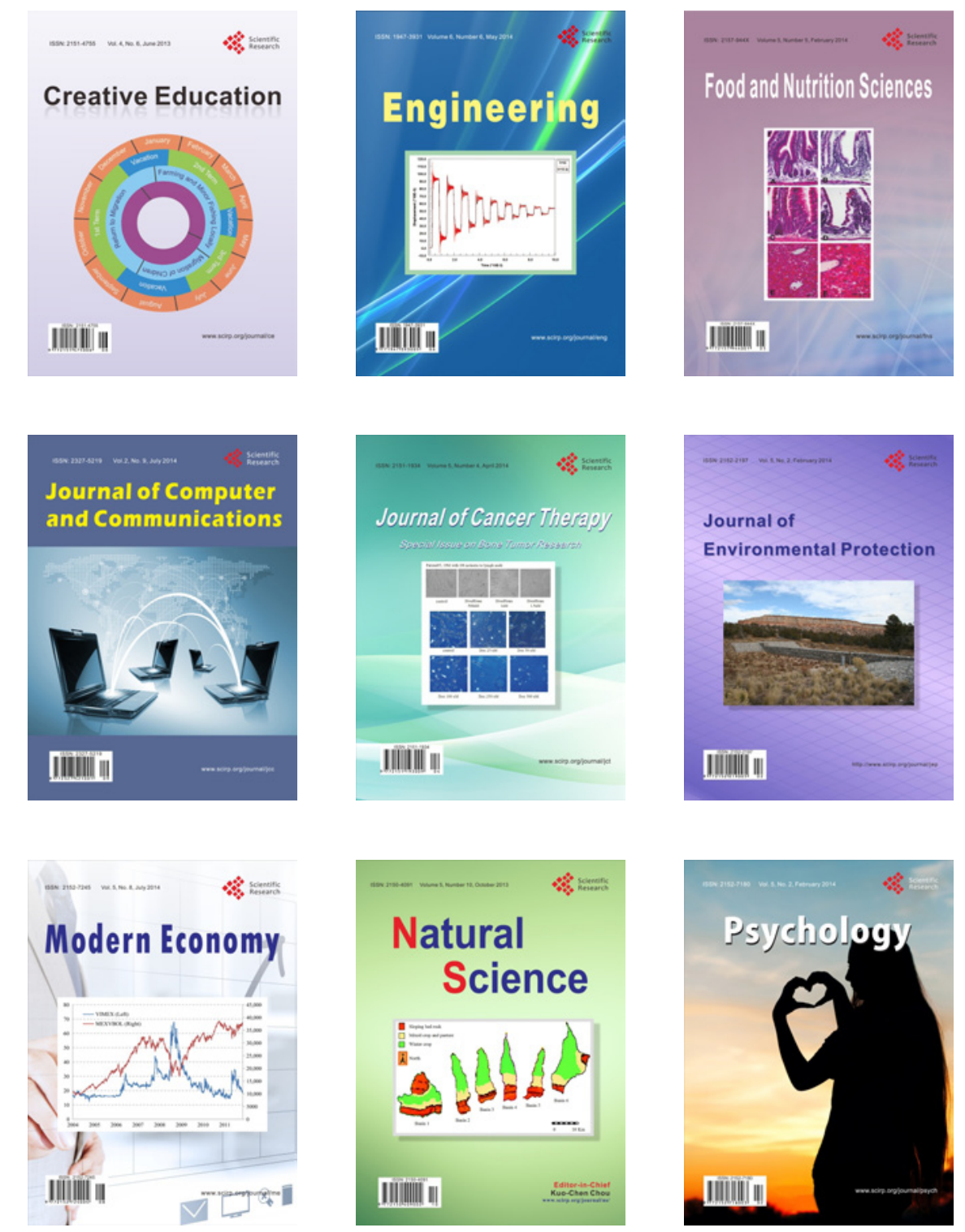